Research

Open Access

\title{
The outcome of extubation failure in a community hospital intensive care unit: a cohort study
}

\author{
Christopher W Seymour ${ }^{1}$, Anthony Martinez ${ }^{2}$, Jason D Christie ${ }^{3}$ and Barry D Fuchs ${ }^{4}$
}

\begin{abstract}
${ }^{1}$ Medical Resident, Hospital of the University of Pennsylvania, Philadelphia, Pennsylvania, USA
2Medical Director, Medical Intensive Care Unit, Division of Pulmonory, and Critical Care, St Agnes Healthcare, Baltimore, Maryland, USA

${ }^{3}$ Assistant Professor of Medicine and Epidemiology, Pulmonory, Allergy and Critical Care Medicine and Center for Epidemiology and Biostatistics, University of Pennsylvania, Philadelphia, Pennsylvania, USA

${ }^{4}$ Medical Director, Medical Intensive Care Unit and Respriatory Care, Hospital of the University of Pennsylvania, Assistant Professor of Medicine,

Pulmonory, Allergy and Critical Care Division, Philadelphia, Pennsylvania, USA
\end{abstract}

Corresponding author: Barry D Fuchs, barry.fuchs@uphs.upenn.edu

Received: 04 February 2004

Revisions requested: 15 March 2004

Revisions received: 14 April 2004

Accepted: 21 June 2004

Published: 20 July 2004
Critical Care 2004, 8:R322-R327 (DOI 10.1186/cc2913)

This article is online at: http://ccforum.com/content/8/5/R322

(c) 2004 Seymour et al.; licensee BioMed Central Ltd. This is an Open Access article: verbatim copying and redistribution of this article are permitted in all media for any purpose, provided this notice is preserved along with the article's original URL.

\begin{abstract}
Introduction Extubation failure has been associated with poor intensive care unit (ICU) and hospital outcomes in tertiary care medical centers. Given the large proportion of critical care delivered in the community setting, our purpose was to determine the impact of extubation failure on patient outcomes in a community hospital ICU.

Methods A retrospective cohort study was performed using data gathered in a 16-bed medical/ surgical ICU in a community hospital. During 30 months, all patients with acute respiratory failure admitted to the ICU were included in the source population if they were mechanically ventilated by endotracheal tube for more than 12 hours. Extubation failure was defined as reinstitution of mechanical ventilation within 72 hours $(n=60)$, and the control cohort included patients who were successfully extubated at 72 hours $(n=93)$.

Results The primary outcome was total ICU length of stay after the initial extubation. Secondary outcomes were total hospital length of stay after the initial extubation, ICU mortality, hospital mortality, and total hospital cost. Patient groups were similar in terms of age, sex, and severity of illness, as assessed using admission Acute Physiology and Chronic Health Evaluation II score $(P>0.05)$. Both ICU (1.0 versus 10 days; $P<0.01)$ and hospital length of stay $(6.0$ versus 17 days; $P<0.01)$ after initial extubation were significantly longer in reintubated patients. ICU mortality was significantly higher in patients who failed extubation (odds ratio $=12.2,95 \%$ confidence interval $[\mathrm{Cl}]=1.5-101 ; P<$ 0.05 ), but there was no significant difference in hospital mortality (odds ratio $=2.1,95 \% \mathrm{Cl}=0.8-5.4$; $P<0.15)$. Total hospital costs (estimated from direct and indirect charges) were significantly increased by a mean of US\$33,926 (95\% Cl = US\$22,573-45,280; $P<0.01)$.

Conclusion Extubation failure in a community hospital is univariately associated with prolonged inpatient care and significantly increased cost. Corroborating data from tertiary care centers, these adverse outcomes highlight the importance of accurate predictors of extubation outcome.
\end{abstract}

Keywords: community hospital, extubation failure, intensive care unit outcome, mechanical ventilation

\section{Introduction}

Approximately $10-15 \%$ of patients who are extubated from mechanical ventilation for acute respiratory failure require reintubation. Compared with patients who are successfully extu- bated, patients who are reintubated have worse clinical outcomes, including prolonged lengths of stay (LOSs) in the intensive care unit (ICU) and hospital, and increased mortality [1-4]. The cause for the increased mortality is not known but 
has been hypothesized to be either a delay in reintubation or a complication of the endotracheal intubation itself. Alternatively, extubation failure may simply be a marker of disease severity, comorbidities, or an unrecognized underlying disease process. When controlling for disease severity using multivariate analysis, recent studies $[5,6]$ found extubation failure to be independently associated with hospital death, although this is not a uniform finding $[7,8]$.

To date, the impact of extubation failure on patient outcomes has been studied exclusively in tertiary care, academic hospitals [1-9]. Because most inpatient care occurs in private, community medical centers, the true scope of the importance of extubation failure to patient outcome remains unknown. Moreover, the hospital costs associated with extubation failure have not been fully explored, having only been reported in postoperative vascular surgery patients [4]. The purpose of the present study was to determine the impact of extubation failure on ICU and hospital mortality, LOS, and total costs in a community hospital ICU.

\section{Methods \\ Study population}

The study was performed by reviewing medical records. The source population included all mechanically ventilated patients admitted to the medical/surgical ICU of a community hospital in Baltimore, Maryland, USA between January 1997 and June 1999 who met the following inclusion criteria: acute respiratory failure as a primary diagnosis; and mechanical ventilation for more than 12 hours. Patients were excluded if they were ventilated noninvasively by mask or via tracheostomy, if extubation occurred inadvertently (unplanned), or if they died or were transferred before extubation. All ICU patients were enrolled in the standardized hospital weaning protocol. Of those in the source population, all patients who failed extubation were included in the study cohort. Of those patients who were successfully extubated, an administrator blinded to the study hypothesis or patient data other than medical record number chose 100 unmatched patients to comprise the control cohort.

\section{Standardized weaning protocol}

Mechanical ventilation was discontinued under the direction of one of three board certified critical care physicians, respiratory therapists, and nursing staff. There was no pulmonary or critical care fellowship program in this ICU, and attending physicians provided off-site coverage at night from home.

A previously established hospital protocol to initiate the weaning process included requirements for hemodynamic stability, improvement in underlying medical conditions, reaching a threshold in three respiratory parameters (i.e. arterial oxygen tension/fractional inspired oxygen ratio $>200$, positive endexpiratory pressure $\leq 5 \mathrm{cmH}_{2} \mathrm{O}$, minute ventilation $\leq 12 \mathrm{l}$ ), and having a satisfactory cough. Patients were evaluated for these criteria as part of a daily screen performed by respiratory therapists. Spontaneous breathing trials were performed using a T-piece and were continued for up to 2 hours if patients maintained a heart rate under 120 beats $/ \mathrm{min}$, pulse oximetry greater than $93 \%$ and respiratory rate under 35 breaths $/ \mathrm{min}$, and had no dysrhythmia, paradoxic breathing, or use of accessory muscles. If spontaneous breathing trials were not tolerated after 2 days, then patients underwent weaning by gradual decrease in pressure support. Patients were considered for extubation if they tolerated T-piece or ventilatory support of no more than $5 \mathrm{cmH}_{2} \mathrm{O}$ continuous positive airway pressure and pressure support of $8 \mathrm{cmH}_{2} \mathrm{O}$ for 2 hours on fractional inspired oxygen under $50 \%$. The decision to extubate was made by the intensivist on duty. Criteria for considering reintubation included, but were not limited to, the same criteria used to evaluate weaning trial tolerance.

\section{Definition of variables}

The primary exposure in this study was extubation failure, which was defined as reinstitution of mechanical ventilation within 72 hours of extubation. Successful extubation was defined as freedom from mechanical ventilation for 72 hours after extubation. The primary outcome was total ICU LOS (in days) after the initial extubation. Secondary outcomes were total hospital LOS (in days) after the initial extubation, ICU mortality, hospital mortality, and total hospital costs and costs per hospital day, which were estimated by abstracting total hospital charges from electronic billing records on all patients, including both direct and indirect charges. Total charges were divided by the institutional charge/cost ratio during the time period of review (1.21): hospital costs per day $=$ (total hospital charges/total hospital length of stay)/1.21 Other outcome data recorded included ICU discharge disposition (step-down unit, floor, or died) and need for tracheostomy during hospitalization.

\section{Demographic data}

Demographic variables were also collected from existing medical records to describe patient groups further. Data obtained included age, sex, and severity of illness by Acute Physiology and Chronic Health Evaluation II score. Etiology of acute respiratory failure was classified as shown in Table 1. The surgical service was recorded as vascular, thoracic, gastrointestinal, orthopedic, or obstetric/gynecologic. Ventilator weaning data obtained included ICU days before the first weaning attempt, and total ventilator days before the first extubation attempt.

\section{Statistical analysis}

Patients who were successfully extubated were compared with the group who failed extubation. Normality of outcome variables was assessed using the Shapiro Wilk test, and results are expressed as mean \pm standard deviation or as median (interquartile range), as appropriate. Either the Student's two-tailed t-test or Wilcoxon rank sum test was used to compare the two groups, depending on normality. Odds 
Table 1

\begin{tabular}{|c|c|}
\hline Classification & Details \\
\hline Pulmonary & $\begin{array}{l}\text { Upper airway obstruction, acute respiratory distress syndrome, chronic obstructuve pulmonary disease, lobar pneumonia, } \\
\text { malignant effusion, aspergilloma, aspiration pneumonitis, lobar collapse, asthma exacerbation, noncardiogenic pulmonary edema }\end{array}$ \\
\hline Cardiac & Congestive heart failure, pericarditis, primary cardiomyopathy, acute myocardial infarction, bacterial endocarditis \\
\hline Neurologic & Status epilepticus, cerebral vascular accident, intracranial hemorrhage, convulsion/seizure disorder \\
\hline Renal & Acute renal failure \\
\hline Other & $\begin{array}{l}\text { Liver failure, drug overdose, upper/lower gastrointestinal bleed, diabetic ketoacidosis, sepsis, blood transfusion reaction, } \\
\text { vasculitis }\end{array}$ \\
\hline
\end{tabular}

ratios (ORs) were calculated using unadjusted logistic regression. Multivariate analysis was not performed because of the limited sample size. $P<0.05$ was considered statistically significant. Statistical analyses were performed using NCSS, version 2000 (NCSS, Kaysville, UT, USA).

\section{Results \\ Demographics}

A total of 1451 mechanically ventilated patients were admitted from January 1997 until June 1999, and 315 patients met eligibility requirements for the study. The principal reason for study exclusion was mechanical ventilation not due to acute respiratory failure as the primary diagnostic category. Of those included, 252 patients were extubated successfully, and 93 of these with available medical records were included in the control group. Study cases were the 60 patients who failed extubation (Fig. 1). As shown in Table 2, patients were similar in terms of age, sex, severity of illness, and etiology of acute respiratory failure, derived from primarily pulmonary causes in both groups. Extubation failure was more common in surgical than in medical patients; of these the gastrointestinal and vascular services were more common in the failed extubation group. Study cases were reintubated a median of 24 hours after extubation.

\section{Outcomes of respiratory failure}

As shown in Table 3, failed extubation was associated with a significant increase in the ICU and hospital LOSs following the initial extubation event ( $P<0.01$ for both); however, total hospital days after ICU discharge were not significantly different between groups. Reintubated patients were more likely to be transferred to a step-down unit, and less likely to be discharged directly to hospital floors. Total charges for hospitalization and cost per day were significantly increased for patients who failed extubation $(P<0.01)$. A higher proportion of patients who failed extubation expired in the ICU (OR = $12.2,95 \%$ confidence interval $=1.5-101 ; P<0.05)$, although hospital mortality of reintubated patients did not reach statistical significance (OR $=2.1,95 \%$ confidence interval $=0.8-$ $5.4 ; P>0.05)$. As shown in Table 4 , the poor outcome follow-
Figure 1

All Mechanically Ventilated (MV) Patients January 1997 - June 1999, $n=1451$

\begin{tabular}{|lll} 
& Non-invasive MV & $n=166$ \\
& Tracheostomy & $n=80$ \\
& MV < 12 hours & $n=130$ \\
& Expired/ withdrawal & $n=156$ \\
Exclude & Unplanned extubation & $n=100$ \\
& $\geq 2^{\circ}$ extubation event & $n=78$ \\
& Transferred off unit & $n=44$ \\
& Primary diagnosis & \\
& not acute resp. failure & $n=382$
\end{tabular}

Patients who met inclusion criteria, $n=315$

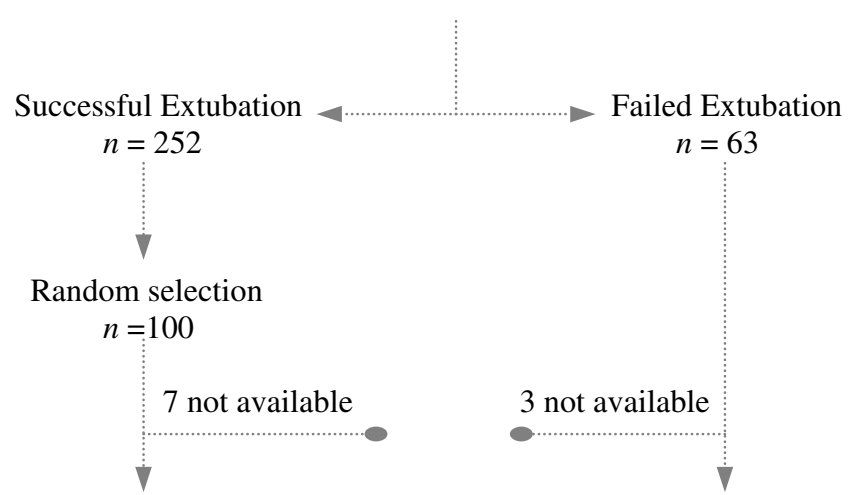

Control cohort $n=93$

Study cohort $n=60$

Methodology for constructing the study.

ing failed extubation was consistent in both subgroups of medical and surgical patients.

\section{Discussion}

This study demonstrates that failed extubation had an adverse impact on clinical outcomes in patients recovering from acute respiratory failure in a community hospital ICU. We found that reintubation increased ICU and hospital LOSs, as well as total hospital costs and cost per hospital day. 
Critical Care October 2004 Vol 8 No 5 Seymour et al.

Table 2

\begin{tabular}{|c|c|c|c|}
\hline Parameters & Total cohort & Successful extubation & Failed extubation \\
\hline$n$ & 153 & 93 & 60 \\
\hline Age (years) & $70(60-78)$ & $70(60-77)$ & $70(59-79)$ \\
\hline Male ( $n[\%])$ & $70(45.8)$ & $37(39.7)$ & $33(55)$ \\
\hline APACHE II score & $21(17-28)$ & $21(17-29)$ & $21(16-26)$ \\
\hline \multicolumn{4}{|l|}{ Etiology of respiratory failure } \\
\hline Pulmonary & $105(68.6)$ & $67(72)$ & $38(63.3)$ \\
\hline Cardiac & $14(9.2)$ & $10(10.8)$ & $4(6.7)$ \\
\hline Neurologic & $10(6.5$ & $5(5.4)$ & $5(8.3)$ \\
\hline Renal & $2(1.3)$ & $2(2.2)$ & $0(0)$ \\
\hline Other & $16(10.5)$ & $6(6.5)$ & $10(16.7)$ \\
\hline Surgical service & $32(20.9)$ & $10(10.8)$ & $22(36.7)$ \\
\hline Gastrointestinal & $15(9.8)$ & $3(3.2)$ & $12(20.0)$ \\
\hline Vascular & $8(5.3)$ & $2(2.2)$ & $6(10.0)$ \\
\hline Thoracic & $6(6.9)$ & $2(2.2)$ & $4(6.7)$ \\
\hline Obstetric/gynecologic & $1(0.6)$ & $1(1.1)$ & $0(0.0)$ \\
\hline Days before initiation of weaning & $1(0.5-2)$ & $1.25(1-2.4)$ & $1(0.5-2)$ \\
\hline Pre-extubation (days) & $3(1-7)$ & $4(2-7)$ & $2(1-5)$ \\
\hline
\end{tabular}

Data presented as median (interquartile range) or as $n$ (\%). APACHE, Acute Physiology and Chronic Health Evaluation.

Table 3

\begin{tabular}{|c|c|c|c|}
\hline Parameters & Successful extubation & Failed extubation & $P$ \\
\hline ICU LOS postextubation (days) & $1(0.3-2)$ & $10(7-15)$ & $<0.01$ \\
\hline \multicolumn{4}{|l|}{ ICU discharge disposition } \\
\hline Step-down unit & $40(43)$ & 35 (58.3) & 0.070 \\
\hline Floor & $48(51.6)$ & $13(21.7)$ & $<0.01$ \\
\hline Died in ICU & $1(1.1)$ & $7(11.7)$ & $<0.01$ \\
\hline Post-ICU LOS (days) & $8.3 \pm 7.3$ & $11.2 \pm 10.7$ & 0.070 \\
\hline Hospital LOS postextubation (days) & $6(4-11)$ & $17(12-23)$ & $<0.01$ \\
\hline Hospital mortality & $9(9.7)$ & $11(18.3)$ & 0.144 \\
\hline Total hospital charges ( $\times$ US $\$ 1000)$ & $23(15-34)$ & $48(34-76)$ & $<0.01$ \\
\hline Cost per hospital day (xUS\$1000) & $1.7 \pm 0.5$ & $2.0 \pm 0.4$ & $<0.01$ \\
\hline Tracheostomy & $7(7.5)$ & $7(11.7)$ & 0.402 \\
\hline Total mechanical ventilation (days) & $4(2-8)$ & $10(6-16)$ & $<0.01$ \\
\hline
\end{tabular}

Data are presented as mean \pm standard deviation, median (interquartile range), or $n(\%)$. ICU, intensive care unit; LOS, length of stay.

In the whole cohort and both medical and surgical subgroups, failed extubation was associated with increased LOS in both the ICU and hospital after the initial extubation (Tables 3 and 4). These findings corroborate data obtained in ICUs at tertiary care centers, and contribute to the available literature by demonstrating the importance of reintubation in the community hospital setting. In an exclusively medical ICU, Epstein and coworkers [6] found that extubation failure prolonged ICU stay 
Table 4

\begin{tabular}{|c|c|c|c|c|c|c|}
\hline \multirow[t]{2}{*}{ Outcomes } & \multicolumn{3}{|c|}{ Medical } & \multicolumn{3}{|c|}{ Surgical } \\
\hline & Successful extubation & Failed extubation & $P$ & Successful extubation & Failed extubation & $P$ \\
\hline$n$ & 83 & 38 & & 10 & 22 & \\
\hline ICU LOS postextubation (days) & $1(0-1)$ & $8(5-15)$ & $<0.01$ & $2(1-4)$ & $12(9-17)$ & $<0.01$ \\
\hline Hospital LOS postextubation (days) & $5(3-10)$ & $15(11-19)$ & $<0.01$ & $6(9-14)$ & $18(16-29)$ & $<0.01$ \\
\hline Hospital mortality (\%) & $8(9.6)$ & $5(13.2)$ & 0.560 & $1(10)$ & $6(27.3)$ & 0.230 \\
\hline Total hospital charges ( $\times$ US $\$ 1000)$ & $26.5 \pm 17.0$ & $49.7 \pm 30.0$ & $<0.01$ & $26.8 \pm 9.0$ & $81.6 \pm 47$ & $<0.01$ \\
\hline
\end{tabular}

Data are presented as mean \pm standard deviation, median (interquartile range) or $n$ (\%). ICU, intensive care unit; LOS, length of stay.

by 17 days after initial extubation, which is greater than the additional 9 days identified in the present study. Similar to our data, Dupont and coworkers [8] showed that reintubation increased ICU LOS by 9 days in an exclusively surgical population. Variations in postextubation failure LOS between populations are known to correlate with the etiology of extubation failure [9], with worse outcomes associated with nonairway etiologies. In addition, complications associated with the process of reintubation, such as ventilator-associated pneumonia, may contribute to the need for prolonged intensive care [10]. These factors were not documented during our review and this is a limitation of our analysis. Failed extubation did not increase post-ICU discharge LOS in this study, despite the increased frequency of transfer to a step-down unit (Table 3); this may be explained by the increased ICU mortality found in reintubated patients.

We found that the increased duration of patient care after failed extubation doubled the total hospital costs and costs per day, as compared with patients who did not require reintubation (Table 3 ). An analysis of the specific constituents that account for this increase in total costs, such as pharmacy, staff, laboratory, or facility expenses, was beyond the scope of the present study. Our findings in a medical/surgical ICU, however, extend the data presented by Pronovost and coworkers [4] from a cohort of vascular surgery patients who failed extubation. They found that reintubation resulted in a $20 \%$ increase in hospital charges. Both reports present hospital charges as a surrogate for hospital costs, which may not be an accurate reflection of actual cost [11].

ICU mortality was significantly higher in patients who failed extubation. This is consistent with the findings of Epstein and Ciubotaru [9], who also reported that sepsis and multiorgan failure were the more common causes of mortality following reintubation. We did not find an association between extubation failure and hospital mortality in our community hospital setting, probably because of limited sample size. This contrasts with findings reported in prior studies performed in some tertiary care centers $[5,6]$, but is similar to the findings of others $[7,8]$. In addition, our hospital mortality rates (Table 3 ) are sub- stantially lower than those reported by Epstein [6] and Esteban [5], which may account for the lack of association between extubation failure and hospital death. The reason for the comparatively higher survival rate of medical patients in our hospital is not clear, but it may be due differences in the etiology or timing of reintubation, because these factors have been shown to have an important influence on patient outcomes $[6,9]$. Alternatively, severity of illness or perhaps some unrecognized aspect of the delivery of patient care, which may differ in the community hospital setting, may also account for these findings.

\section{Conclusion}

We have demonstrated that extubation failure may be an important ICU complication because of its association with adverse patient outcomes and cost in a community hospital. Through univariate analysis, we corroborated the unfavorable consequences of reintubation, such as increased ICU and hospital LOSs, that were previously reported in tertiary care academic hospitals. Our findings underscore the need for further study of predictive indices of extubation outcome that may help to prevent the substantial morbidity associated with reintubation.

\section{Key messages}

- Failed extubation is associated with adverse patient outcomes in a community hospital.

- Reintubation increased ICU mortality, hospital cost, and ICU and hospital LOSs after extubation.

- These findings are consistent with the poor outcome following failed extubation in tertiary care centers.

\section{Competing interests}

None declared. 


\section{Acknowledgements}

We would like to thank Christina Gaughan, MS, of the University of Pennsylvania Center for Clinical Epidemiology and Biostatistics for her advice and expertise, and Donna Casella and Diane Alberter for their assistance with manuscript preparation.

\section{References}

1. Esteban A, Frutos F, Tobin M, Alia I, Solsona J, Valverdu I, Fernandez R, De la Cal JF, Benito S, Tomas $\mathrm{R}$ et al.: A comparison of four methods of weaning patients from mechanical ventilation. N Engl J Med 1995, 332:345-352.

2. Ely E, Baker A, Dunagon D, Burke HL, Smith AC, Kelly PT, Johnson MM, Browder RW, Bowton DL, Haponik EF: Effect on duration of mechanical ventilation of identifying patient capable of breathing spontaneously. N Engl J Med 1996, 335:1864-1869.

3. Vallverdu I, Calaf N, Subirana M, Net A, Benito S, Mancebo J: Clinical characteristics, respiratory functional parameters and outcome of a 2-hour T-piece trial in patients weaning from mechanical ventilation. Am J Respir Crit Care Med 1998, 158:1855-1862.

4. Pronovost PJ, Garrett E, Dorman T, Jenckes M, Webb TH III, Breslow $M$, Rosenfeld $B$, Bass $E$ : Variations in complication rates and opportunities for improvement in quality of care for patients having abdominal aortic surgery. Lang Arch Surg 2001, 386:249-256.

5. Esteban A, Alia I, Gordo F, Fernandez R, Solsona JF, Vallverdu I, Macias S, Allegue JM, Blanco J, Carriedo D et al.: Extubation outcome after spontaneous breathing trials with T-tube or pressure support ventilation. The Spanish Lung Failure Collaborative Group. Am J Respir Crit Care Med 1997, 156:459-465.

6. Epstein S, Ciubotaru R, Wong J: Effect of failed extubation on the outcome of mechanical ventilation. Chest 1997, 112:186-192.

7. Rady MY, Ryan T: Perioperative predictors of extubation failure and the effect on clinical outcome after cardiac surgery. Crit Care Med 1999, 27:340-347.

8. Dupont H, LePort Y, Paugham-Burtz C, Mantz J, Desmonts M: Reintubation after planned extubation in surgical ICU patients: a case-control study. Intensive Care Med 2001, 27:1875-1880.

9. Epstein SK, Ciubotaru RL: Independent effects of etiology of failure and time to reintubation on outcome for patients failing extubation. Am J Respir Crit Care Med 1998, 158:489-493.

10. Torres A, Gatell J, Aznar E, El-Ebiary M, Puig de la Bellacasa J, Gonzalez J, Ferrer M, Rodriguez-Roisin R: Re-intubation increases the risk of nosocomial pneumonia in patients needing mechanical ventilation. Am J Respir Crit Care Med 1995, 152:137-141.

11. Pines JM, Fager SS, Milzman DP: A review of costing methodologies in critical care studies. J Crit Care 2002, 17:181-186. 